\title{
Antiretroviral Treatment-Associated Hepatotoxicity and Anemia in Patients Receiving Stavudine or Zidovudine Containing Regimens in Sub- Saharan African Settings
} \author{
Stefano Orlando ${ }^{2}$, Leonardo Palombi ${ }^{2}$ and Giuseppe Liotta ${ }^{2}$ \\ ${ }^{1}$ Community of Sant'Egidio, DREAM Program, Wuerzburg, Germany \\ ${ }^{2}$ Department of Biomedicine and Prevention, University of Tor Vergata; Community of Sant'Egidio, DREAM Program; Rome, Italy \\ ${ }^{3}$ Community of Sant'Egidio, DREAM Program, Maputo, Mozambique \\ ${ }^{4}$ Community of Sant'Egidio, DREAM Program, Blantyre, Malawi \\ ${ }^{5}$ Department of Preventive Medicine, LUMSA University, Community of Sant'Egidio, DREAM Program, Rome, Italy
}

Dieter Wenderlein ${ }^{1 *}$, Paola Scarcella ${ }^{2}$, Ines Zimba ${ }^{3}$, Richard Luhanga ${ }^{4}$, Sandro Mancinelli ${ }^{2}$, Maria Cristina Marazzi ${ }^{5}$, Ersilia Buonomo²,

\begin{abstract}
Objective: Purpose of this study was to assess the prevalence and risk factors associated with development of hepatotoxicity and anemia following initiation of antiretroviral treatment (ART) containing stavudine (d4T) or zidovudine (AZT) in the first year of treatment in the African setting.

Method: We evaluated aspartate aminotransferase and haemoglobin levels at baseline and at 1, 2, 3, 6, 9 and 12 months following ART initiation among 10,537 HIV-1 infected, ART-naïve, non-pregnant adults in Mozambique and Malawi. The Cox proportional hazards model was used to assess risk factors for hepatotoxicity and anaemia in the first year following ART initiation.

Results: The prevalence of ART-associated hepatotoxicity grades 1-2 declined in the first 3 months of ART from $13.5 \%$ to $10.8 \%$, and grades $3-4$ from $2.0 \%$ to $0.2 \%$ from month 1 to month 6 . The prevalence of hepatotoxicity grades 1-2 peaked at month 6 due to the use of d4T (overall $14.2 \%$; d4T-arm: $16.2 \%$, AZT-arm: $5.8 \%$ ). Anemia grades $1-2$ and 3-4 declined from month 1 (13.3\%, 3.2\% respectively) to month 12 (3.0\%, $0.5 \%$, respectively).

Risk factors for hepatotoxicity grades 1-2 included d4T use, an elevated VL pre-ART and female sex, and for anemia grade 1-2 and 3-4 AZT use, female sex and malaria. A high pre-ART VL was associated with the onset of severe anaemia. Not being malnourished was protective against mild hepatotoxicity and anemia. The ART-related mortality observed in the cohort was low $(0.017 \%)$.

Conclusion: In African settings the risk of untreated HIV outweighs the risk of anemia and hepatotoxicity mediated by ART. The prevalence of ART-mediated hepatotoxicity declines in the first 3 to 6 months of treatment, and that of anemia declines in the first 12 months of ART. Patients with a poor health status at the start of ART are at highest risk of developing ART-associated hepatotoxicity and anemia.
\end{abstract}

Keywords: HIV-1; Antiretroviral therapy; Sub-Saharan Africa; Adverse effects; Hepatotoxicity; Anemia; Malaria; Risk factors

\section{Background}

In an era of rapid expansion of antiretroviral treatment (ART) availability in sub-Saharan Africa, the management of ART-mediated toxicities is becoming increasingly important. ART-associated hepatotoxicity and anemia is known causes of morbidity, mortality and treatment interruption [1-4], but the association and timing for development of hepatotoxicity, anemia, HIV-infection and ART in the African population is not well characterized.

Low-grade elevations of liver-enzymes may occur with every ART combination. They are frequent, mostly asymptomatic and often resolve spontaneously, but may be associated with severe and potentially life-threatening clinical manifestations. In studies from sub-Saharan Africa, the risk of ART-associated hepatotoxicity ranges from $1.0 \%$ [5] to $17 \%$ [6] depending on the ART combination, the definition of hepatotoxicity and characteristics of the study population. Stavudine (d4T) and nevirapine (NVP) are the antiretroviral drugs (ARVs) with the highest hepatotoxic potential [2,7-9].

Anemia is the most common hematologic abnormality in HIV infected persons. There is a significant and graded association between anemia and the progression to AIDS and death $[4,10,11]$. Anemia at the beginning of ART is an important parameter for the short and long- term prognosis [4]. ART generally improves HIV-associated anemia $[3,4]$, but some ARVs may cause anemia. Zidovudine (AZT) is the ARV with the highest myelosuppressive potential, causing anemia in $5 \%$ of patients $[12,13]$

D4T, AZT and nevirapine (NVP) are still widely used in sub-Saharan Africa, even though the current WHO Guidelines [14] recommend $\mathrm{TDF}+3 \mathrm{TC} / \mathrm{FTC}+\mathrm{EFV}$ as preferred first-line combinations. Knowledge regarding side effects of ART in the African setting is limited. In order to evaluate hepatotoxicity and anemia as the most relevant $\mathrm{d} 4 \mathrm{~T}$-, AZTand NVP-associated adverse effects; we analyzed the prevalence of elevated liver-enzyme values (aspartate aminotransferase, AST) and

*Corresponding author: Dieter Wenderlein, Community of Sant'Egidio DREAM Program, Wuerzburg, Germany, Tel: +491704336394; E-mail: dieterwenderlein@santegidio.de

Received November 13, 2015; Accepted January 13, 2016; Published January 20, 2016

Citation: Wenderlein D, Scarcella P, Zimba I, Luhanga R, Mancinelli S, et al (2016) Antiretroviral Treatment-Associated Hepatotoxicity and Anemia in Patients Receiving Stavudine or Zidovudine Containing Regimens in Sub-Saharan African Settings. J AIDS Clin Res 7: 537. doi:10.4172/2155-6113.1000537

Copyright: (C) 2016 Wenderlein D, et al. This is an open-access article distributed under the terms of the Creative Commons Attribution License, which permits unrestricted use, distribution, and reproduction in any medium, provided the original author and source are credited. 
Citation: Wenderlein D, Scarcella P, Zimba I, Luhanga R, Mancinelli S, et al. (2016) Antiretroviral Treatment-Associated Hepatotoxicity and Anemia in Patients Receiving Stavudine or Zidovudine Containing Regimens in Sub-Saharan African Settings. JAIDS Clin Res 7: 537. doi:10.4172/21556113.1000537

Page 2 of 8

decreased hemoglobin (HB) as well as risk factors associated with these findings during the first year of ART in a huge cohort of patients from Mozambique and Malawi.

\section{Method}

\section{Study design}

Multicenter retrospective observational cohort study.

\section{Study population}

The inclusion criteria included infection with HIV-1, age 15 years or older, being ART-naïve with the indication to start ART according to clinical, immunological and/or virological criteria, and informed consent to participate in the program. Patients who did not complete the first year of ART because they died or were lost-to-follow up were excluded from the analysis as well as patients with elevated AST and $\mathrm{Hb}$ levels at the baseline visit. All patients from four AIDS-treatment sites of the DREAM Program in Mozambique (one urban site in Maputo, three sites in the outskirts of Maputo, Matola and Beira) and three in Malawi (one urban site in Blantyre, two rural sites outside Lilongwe and Balaka) who fulfilled the inclusion criteria were enrolled in the study.

The DREAM Program is run by the Community of Sant'Egidio in ten African countries. It offers a comprehensive, free-of-charge package of ART, prevention of mother-to-child transmission (PMTCT), laboratory diagnostics including CD4 count and plasma HIV RNA viral load (VL), food integration and other services. Agreements with the Ministries of Health regulate the implementation of AIDS treatment sites including operational research.

Hepatitis B (HBV) and C (HCV) infectionas well as Tuberculosis (TB) co-infection were not assessed in this specific analysis. Therefore, HIV patients with HBV, HCV and TB co-infection and on TB-treatment are included in the cohort.

In the final analysis we included only patients without any abnormal AST and HB values before ART initiation, in order to evaluate ARTassociated hepatotoxicity and anemia.

\section{Method}

Data for all patients, who started ART from 01.01.2006 to 31.08.2011 in Mozambique and until 31.12.2009 in Malawi, were reviewed. The follow-up period was until 12 months after the initiation of ART. ART was started in patients with a WHO stage 3 or 4 , or a CD 4 count $<350$ cells/ $\mu \mathrm{l}$, or a CD 4 count between 350 and 500 cells/ $\mu \mathrm{l}$ and VL $>5 \log _{10}$ $\mathrm{c} / \mathrm{ml}$.

Initial first-line ART regimens consisted of d4T- and AZT-based combinations; $95 \%$ of the patients received NVP as the NNRTI of choice. At the time of study initiation, the national guidelines of Mozambique and Malawi recommended as the first line regimen $\mathrm{d} 4 \mathrm{~T}+3 \mathrm{TC}+\mathrm{NVP}$. In June 2010, d4T was substituted in Mozambique by AZT as a first line regimen. In the case of severe peripheral neuropathy, $\mathrm{d} 4 \mathrm{~T}$ was replaced by AZT. In the case of hepatotoxicities or severe skin reactions NVP was substituted by EFV or LPV/r.

\section{Variables collected and outcome definitions}

AST and $\mathrm{HB}$ values were graded according to the table for grading the severity of adult and pediatric adverse events of the AIDS Clinical Trials Group [15]. Mild and moderate hepatotoxicity (grade 1-2) was defined as AST 1.1-5.0 U/l $\times$ Upper limit of normal (ULN), and severe and potentially life threatening hepatotoxicity (grade 3-4) as AST $>5.1$ $\mathrm{U} / \mathrm{l} \times \mathrm{ULN}$. ULN for females was $35 \mathrm{U} / \mathrm{l}$ and for males $50 \mathrm{U} / \mathrm{l}$. Mild and moderate anemia (grade 1-2) was HB 7.5-10.0 g/dl, and severe and potentially life threatening anemia (grade $3-4$ ) was $\mathrm{HB}<7.5 \mathrm{~g} / \mathrm{dl}$.

Baseline CD4 count, VL, BMI, AST and HB were measured before starting ART. AST and HB were measured at 1, 2, 3, 6, 9 and 12 months after start of ART. Prevalence of hepatotoxicity grades 1-2 and 3-4 and of anemia grades 1-2 and 3-4 were analyzed both in the complete cohort and in the two arms with d4T-based and AZT-based regimens. These potential risk factors were assessed by univariate and multivariate analysis: type of NRTI (d4T vs. AZT), type of NNRTI (NVP- vs. EFV), CD4 count before ART stratified in CD4 $\leq 250$, CD4 251-399 and CD4 $\geq 400$, VL before ART (VL $\leq 5 \log _{10}$ copies/ $\mu \mathrm{l}$ vs. VL $>5 \log _{10}$ copies $/ \mu \mathrm{l}$ ), nutritional status before ART (BMI), sex and cumulative malaria events. Patients with a BMI below 18.5 were considered as malnourished. Cumulative malaria was defined as at least one malaria attack after the start of ART which was confirmed by a rapid diagnostic test.

\section{Statistical analysis}

SPSS v.Win 19.0 (SPSS Inc., Chicago, Illinois, USA) was used for statistical data analysis. Relative risk (RR) ninety-five percent confidence intervals and $p$-values were calculated. To assess risk factors for hepatotoxicity and anemia in the d4T- and AZT-arms, data were dichotomized according to pre-ART CD4 cell counts, VL, BMI and $\mathrm{HB}$ in order to generate relative risk values for each stratum. Risk factors were identified by univariate and multivariate analyses (Cox proportional hazards model).

\section{Results}

Medical files of all 11,753 adult, ART naïve, non-pregnant patients, initiating ART were reviewed. Twelve months after ART initiation, $744(6.3 \%)$ of patients had died and $442(3.8 \%)$ were lost to followup. The 1186 patients $(10.1 \%)$ who did not complete the first year of ART, and $30(0.3 \%)$ who did not receive d4T- or AZT-based regimens were excluded. From the remaining 10,537 patients, 6804 (64.4\%) were female and 3733 (35.4\%) male. From 10,434 patients with available CD4 counts, $6469(62.0 \%)$ had a CD4 $\leq 250,3193$ (30.6\%) a CD4 251399 , and $772(7.4 \%)$ a CD4 $\geq 400.8231(78.1 \%)$ received a d4T-based and $2306(21.9 \%)$ an AZT-based combination. Only $6(0.06 \%)$ patients did not take lamivudine (3TC) as the second line NRTI (1 didanosine, 5 abacavir). 10,043 (95.3\%) received NVP as the NNRTI of choice and 254 (2.4\%) EFV; 240 patients (2.3\%) did not take a NNRTI as a third component (191 abacavir, 23 indinavir, 22 lopinavir/r, 4 nelfinavir).

\begin{tabular}{|c|c|c|c|c|c|}
\hline Pre-ART values & d4T-based arm & AZT-based arm & Missing values & $\mathrm{T}$ test $(\mathrm{Cl} 95 \%)$ & $\mathbf{P}$ \\
\hline Age in years $(n)$ & $36.22(8231)$ & $34.41(2306)$ & - & $1.81(1.33-2.28)$ & $<0.001$ \\
\hline 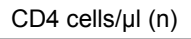 & $239.38(8151)$ & $333.23(2283)$ & 103 & $93.85(82.83-104.86)$ & $<0.001$ \\
\hline $\mathrm{VL} \log _{10} \mathrm{c} / \mathrm{ml}(\mathrm{n})$ & 4.93 (7798) & $4.71(2203)$ & 536 & & $>0.001$ \\
\hline BMI (n) & $20.71(8201)$ & $22.22(2297)$ & 39 & $1.51(1.31-1.71)$ & $<0.001$ \\
\hline $\mathrm{HB}$ g/dl (n) & $10.91(8173)$ & $11.32(2283)$ & 81 & $0.41(0.19-0.62)$ & $<0.001$ \\
\hline
\end{tabular}

Table 1: Pre-ART characteristics by treatment arm. 
Citation: Wenderlein D, Scarcella P, Zimba I, Luhanga R, Mancinelli S, et al. (2016) Antiretroviral Treatment-Associated Hepatotoxicity and Anemia in Patients Receiving Stavudine or Zidovudine Containing Regimens in Sub-Saharan African Settings. JAIDS Clin Res 7: 537. doi:10.4172/21556113.1000537

Page 3 of 8

Baseline CD4 count, BMI and Hb values were significantly different between patients receiving AZT-based and d4T-based ART regimens (Table 1). Overall, patients in the AZT-arm were younger and in better health than those in the d4T-arm. However, baseline differences did not seem to affect further analysis.

The baseline prevalence of hepatotoxicity grades 1-2 and 3-4 and of anemia grades $1-2$ and 3-4 were $18.1 \%$ (1515/8381), 0.5\% (38/8381), $28.5 \%(2599 / 9131)$ and $6.7 \%(610 / 9131)$, respectively.

\section{ART-associated hepatotoxicity during the first year of ART}

The rates of ART-associated hepatotoxicity 1-2 declined from $13.5 \%$ to $10.8 \%$ in the total cohort in the first 3 months of ART, peaked in month 6
(14.2\%) and remained stable in month 9 (9.6\%) and 12 (10.0\%) (Table 2, Figures 1 and 2). The prevalence of hepatotoxicity grades 1-2 during the first year of ART was higher in the d4T- than the AZT-arm. The differences between grades 1-2 liver toxicity among the two arms was 5.5\% after the first month, reached a maximum difference of $10.4 \%$ after 6 months and then diminished to a minimum value of $3.1 \%$ after 12 months. At 6 months the prevalence of hepatotoxicity peaked in the $\mathrm{d} 4 \mathrm{~T}$-arm (16.2\%), whilst in the AZT-arm it reached its lowest level at 5.8\%.

The rate of hepatotoxicity grades 3-4 in the total cohort decreased from $2.0 \%$ after the first month to $0.2 \%$ after month 6 . In the d4T-arm it dropped from $1.9 \%$ to $0.6 \%$ in the first two months, and in the AZTarm it decreased from $2.3 \%$ to $0.5 \%$ until month 3 . Then hepatotoxicity

\begin{tabular}{|c|c|c|c|c|c|c|}
\hline \multirow[t]{2}{*}{ Month } & \multirow{2}{*}{$\begin{array}{c}\begin{array}{c}\text { Prevalence of } \\
\text { hepatotoxicity } 1-2\end{array} \\
\text { Total }\end{array}$} & \multirow{2}{*}{$\begin{array}{c}\begin{array}{c}\text { Prevalence of } \\
\text { hepatotoxicity 3-4 }\end{array} \\
\text { Total }\end{array}$} & \multicolumn{2}{|c|}{ Prevalence of hepatotoxicity $1-2$} & \multicolumn{2}{|c|}{ Prevalence of hepatotoxicity 3-4 } \\
\hline & & & d4T-based arm & AZT-based arm & d4T-based arm & AZT-based arm \\
\hline Month 1 & $13.5 \%(613 / 4544)$ & $2.0 \%(119 / 5893)$ & $14.6 \%(530 / 3634)$ & $9.1 \%(83 / 910)$ & $1.9 \%(93 / 4776)$ & $2.3 \%(26 / 1117)$ \\
\hline Month 2 & $11.1 \%(390 / 3510)$ & $0.8 \%(35 / 4583)$ & $12.0 \%(338 / 2805)$ & $7.4 \%(52 / 705)$ & $0.6 \%(24 / 3718)$ & $1.3 \%(11 / 865)$ \\
\hline Month 3 & $10.8 \%(475 / 4396)$ & $0.5 \%(30 / 5733)$ & $11.8 \%(420 / 3567)$ & $6.6 \%(55 / 829)$ & $0.5 \%(25 / 4710)$ & $0.5 \%(5 / 1023)$ \\
\hline Month 6 & $14.2 \%(580 / 4084)$ & $0.2 \%(9 / 5268)$ & $16.2 \%(534 / 3287)$ & $5.8 \%(46 / 797)$ & $0.2 \%(7 / 4296)$ & $0.2 \%(2 / 972)$ \\
\hline Month 9 & $9.6 \%(187 / 1951)$ & $0.3 \%(7 / 2485)$ & $11.5 \%(147 / 1280)$ & $6.0 \%(40 / 671)$ & $0.2 \%(4 / 1668)$ & $0.4 \%(3 / 817)$ \\
\hline Month 12 & $10.0 \%(242 / 2422)$ & $0.4 \%(12 / 3124)$ & $10.7 \%(202 / 1893)$ & $7.6 \%(40 / 529)$ & $0.4 \%(9 / 2473)$ & $0.5 \%(3 / 651)$ \\
\hline
\end{tabular}

Table 2: Prevalence of ART-associated hepatotoxicity grades 1-2 and 3-4.

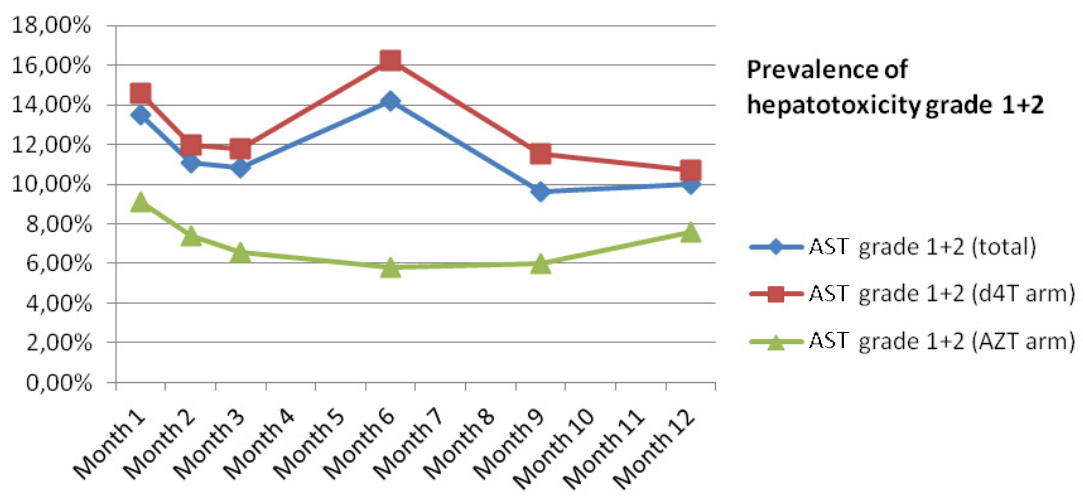

Figure 1: Prevalence of ART-associated hepatotoxicity grades 1-2

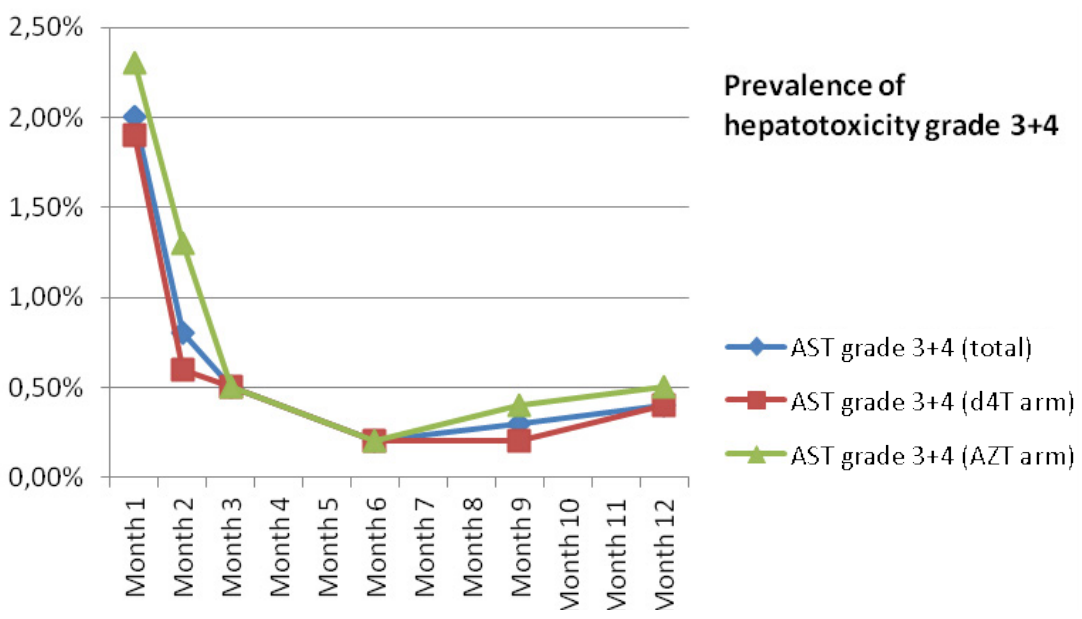

Figure 2: Prevalence of ART-associated hepatotoxicity grades 3-4. 
Citation: Wenderlein D, Scarcella P, Zimba I, Luhanga R, Mancinelli S, et al. (2016) Antiretroviral Treatment-Associated Hepatotoxicity and Anemia in Patients Receiving Stavudine or Zidovudine Containing Regimens in Sub-Saharan African Settings. JAIDS Clin Res 7: 537. doi:10.4172/21556113.1000537

Page 4 of 8

levels remained below $1 \%$ in both arms until month 12. A higher prevalence of liver toxicity grades $3-4$ in the $\mathrm{d} 4 \mathrm{~T}$ - versus the AZT-arm was not observed.

\section{Risk factors for hepatotoxicity using d4T- and AZT-based regimens}

In the univariate analysis for $\mathrm{d} 4 \mathrm{~T}$, a baseline CD4 count below 250/ $\mu \mathrm{l}$, a pre-ART VL>5 $\log _{10} \mathrm{c} / \mathrm{ml}$ and female gender were associated with a significant risk for hepatotoxicity grades 1-2 (Table 3). Multivariate analysis confirmed these risk factors except for the CD4 count. Not being malnourished (BMI>18.5) before ART was a protective risk factor against mild to moderate liver toxicity both in the univariate and multivariate analysis. The univariate and multivariate analysis did not find any significant associations for development of grades 3-4 liver toxicity.

\begin{tabular}{|c|c|c|c|c|}
\hline \multirow[t]{3}{*}{ Variable } & \multirow{2}{*}{\multicolumn{2}{|c|}{$\begin{array}{l}\text { Univariate analysis } \\
\text { Hazard ratio }(95 \% \mathrm{Cl})\end{array}$}} & \multirow{2}{*}{\multicolumn{2}{|c|}{$\begin{array}{l}\text { Multivariate analysis } \\
\text { Hazard ratio }(95 \% \mathrm{Cl})\end{array}$}} \\
\hline & & & & \\
\hline & Hepatotoxicity 1-2 & Hepatotoxicity 3-4 & Hepatotoxicity 1-2 & Hepatotoxicity 3-4 \\
\hline NRTI (d4T- vs. AZT) & $1.78(1.56-2.02)$ & Non-significant & $1.83(1.54-2.16)$ & Non-significant \\
\hline Pre-ART CD4+ cell count $(\leq 250 / \mu l$ vs $>250 / \mu l)$ & $1.25(1.05-1.49)$ & Non-significant & Non-significant & Non-significant \\
\hline Pre-ART VL (>5.0 Log vs < 5.0 Log) & $1.22(1.01-1.36)$ & Non-significant & $1.22(1.08-1.39)$ & Non-significant \\
\hline Pre-ART BMI (<18.5 vs. >18.6) & $0.78(0.69-0.88)$ & Non-significant & $0.79(0.70-0.89)$ & Non-significant \\
\hline gender (female vs. male) & $1.18(1.12-1.24)$ & Non-significant & $1.47(1.30-1.66)$ & Non-significant \\
\hline
\end{tabular}

Table 3: Cox proportional hazards analysis of factors associated with increased ART-associated hepatotoxicity grades 1-2 and 3-4.

\begin{tabular}{|c|c|c|c|c|c|c|}
\hline \multirow{2}{*}{ Month } & \multirow{2}{*}{$\begin{array}{c}\text { Prevalence of anemia } \\
1-2 \\
\text { Total }\end{array}$} & \multirow{2}{*}{$\begin{array}{c}\text { Prevalence of anemia } \\
3-4 \\
\text { Total }\end{array}$} & \multicolumn{2}{|c|}{ Prevalence of anemia 1-2 } & \multicolumn{2}{|c|}{ Prevalence of anemia 3-4 } \\
\hline & & & d4T-based arm & AZT-based arm & d4T-based arm & AZT-based arm \\
\hline Month 1 & $13.3 \%(631 / 4737)$ & $3.2 \%(226 / 7021)$ & $12.1 \%(456 / 3766)$ & $18.0 \%(175 / 971)$ & $3.2 \%(180 / 5551)$ & $3.1 \%(46 / 1470)$ \\
\hline Month 2 & $9.3 \%(310 / 3334)$ & $2.1 \%(102 / 4808)$ & $7.5 \%(200 / 2675)$ & $16.7 \%(110 / 659)$ & $1.8 \%(69 / 3834)$ & $3.4 \%(33 / 974)$ \\
\hline Month 3 & $7.0 \%(299 / 4265)$ & $1.5 \%(92 / 6194)$ & $5.8 \%(202 / 3459)$ & $12.0 \%(97 / 806)$ & $1.1 \%(53 / 4981)$ & $3.2 \%(39 / 1213)$ \\
\hline Month 6 & $4.8 \%(193 / 3993)$ & $0.8 \%(48 / 5715)$ & $4.3 \%(138 / 3188)$ & $6.8 \%(55 / 805)$ & $0.6 \%(27 / 4539)$ & $1.8 \%(21 / 1176)$ \\
\hline Month 9 & $4.0 \%(129 / 3233)$ & $0.7 \%(33 / 4595)$ & $3.5 \%(89 / 2555)$ & $5.9 \%(40 / 678)$ & $0.7 \%(24 / 3601)$ & $0.9 \%(9 / 994)$ \\
\hline Month 12 & $3.0 \%(73 / 2454)$ & $0.5 \%(19 / 3511)$ & $2.3 \%(44 / 1941)$ & $5.7 \%(29 / 513)$ & $0.5 \%(14 / 2760)$ & $0.7 \%(5 / 751)$ \\
\hline
\end{tabular}

Table 4: Prevalence of ART-associated anemia grades 1-2 and 3-4.

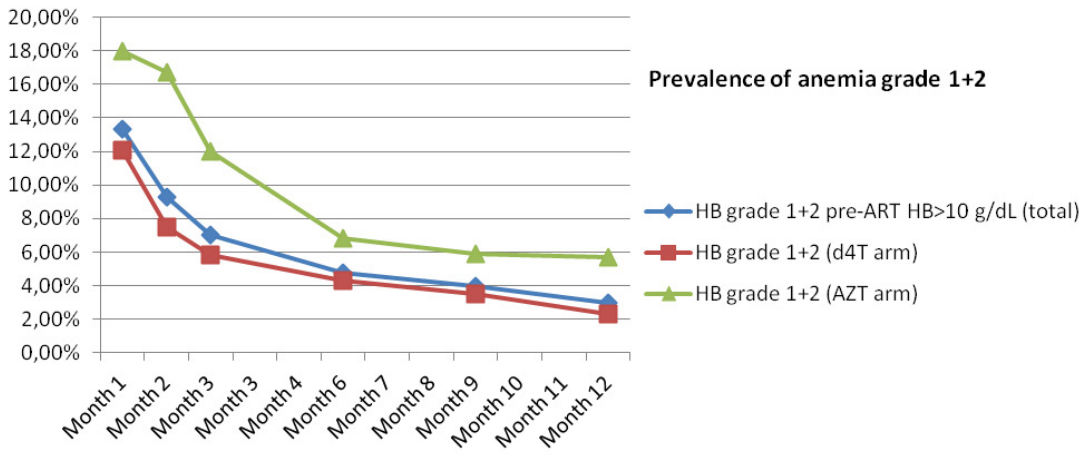

Figure 3: Prevalence of ART-associated anemia grades 1-2.

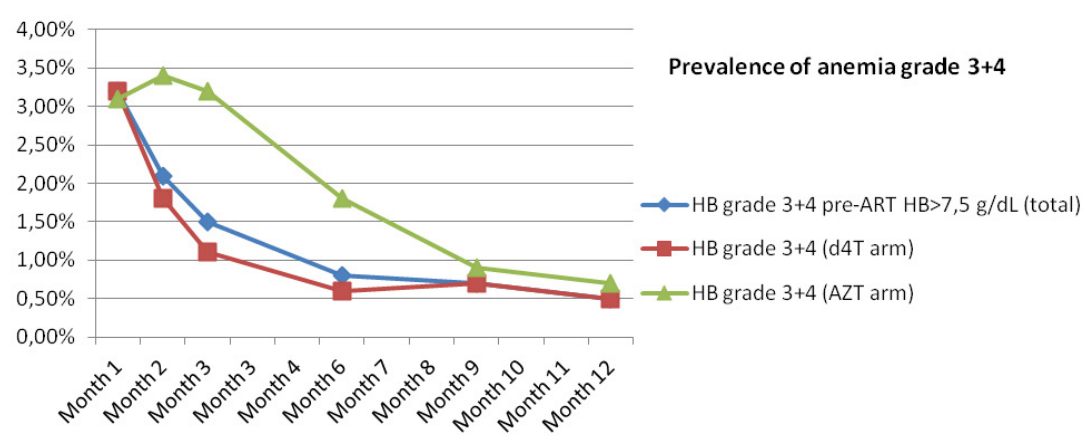

Figure 4: Prevalence of ART-associated anemia grades 3-4. 
Citation: Wenderlein D, Scarcella P, Zimba I, Luhanga R, Mancinelli S, et al. (2016) Antiretroviral Treatment-Associated Hepatotoxicity and Anemia in Patients Receiving Stavudine or Zidovudine Containing Regimens in Sub-Saharan African Settings. J AIDS Clin Res 7: 537. doi:10.4172/21556113.1000537

Page 5 of 8

\section{ART-associated anemia during the first year of ART}

The prevalence of ART-related mild and moderate anemia in the cohort was $13.3 \%$ after 1 month and decreased continuously to a prevalence of $3.0 \%$ after one year (Table 4 , Figures 3 and 4 ). During the first year of ART, the prevalence of anemia grades 1-2 was persistently higher in patients who received AZT as compared to d4T. The highest difference in prevalence of anemia grades $1-2$ of $9.2 \%$ after 2 months of ART initiation between the AZT- and d4T-arms decreased to $2.4 \%$ after 9 months.

The rate of severe and potentially life threatening anemia in the cohort decreased from $3.2 \%$ in month 1 to $0.5 \%$ after 12 months; it remained below 1\% from month 6 to 12 . Anemia grades 3-4 was more frequent in the AZT- than in the d4T-arm in all months, except a marginal difference after month 1 (AZT-arm: 3.1\%, d4T-arm: $3.2 \%$ ). The difference of anemia grades 3-4 between the AZT- and d4T-arms was $2.1 \%$ in month 3 , and decreased to a minimum difference of $0.2 \%$ in months 9 and 12. In contrast to the continuously declining curve of anemia grades 1-2 in the AZT-arm, the prevalence of AZT-associated anemia grades 3-4 was quite stable during the first 3 months (month 1: $3.1 \%$, month $2: 3.4 \%$, month $3: 3.2 \%)$. This suggests that anemia grades 3-4 (but not anemia grades 1-2) induced by AZT in the first three months was stronger than the anemia reverting effect of ART.

\section{Risk factors for anemia with $\mathrm{d} 4 \mathrm{~T}$ - and AZT-based regimens}

In an univariate and multivariate analysis, AZT and cumulative malaria were the strongest risk factors for anemia grades 1-2 and 3-4 (Table 5). Female gender was a predictor only for mild and moderate anemia, and a pre-ART VL $>5 \log _{10} \mathrm{c} / \mathrm{ml}$ was a risk factor for severe and life threatening anemia. A good nutritional status (BMI>18.5) before ART protected against anemia of any grade. Interestingly no CD4 stratum $(C D 4 \leq 250$, CD4 251-399, CD4 $\geq 400$ ) was a significant risk factor for anemia.

\section{ART-toxicity related deaths}

Two deaths among 744 patients who died were clearly associated with antiretroviral toxicity: A 45 years old male patient on AZT $+3 \mathrm{TC}+\mathrm{NVP}$ died of megaloblastic anemia after 3.5 months of ART. Another male patient, age 32 years, who received $\mathrm{d} 4 \mathrm{~T}+3 \mathrm{TC}+\mathrm{NVP}$, died of acute hepatitis after nearly 2 months of ART. ART-associated mortality was very low at $0.017 \%(2 / 11,753)$.

\section{Cumulative malaria}

Because anemia is a common complication of all types of malaria, we assessed cumulative malaria as a risk factor of anemia in the multivariate analysis. Malaria-related anemia was significantly associated with increased morbidity and mortality, especially in children and pregnant women $[16,17]$. The prevalence of malaria was
$5.5 \%$ after 1 month of ART, decreased quickly in the second (1.7\%) and third months of therapy (1.5\%), peaked in month $6(2.9 \%)$ and $9(2.4 \%)$ and decreased again to $1.6 \%$ after month 12 . Cumulative Malaria was the strongest risk factor for severe and life threatening anemia in the multivariate analysis, and the second strongest risk factor after AZT in the univariate analysis: At least one malaria attack after the initiation of ART quadrupled the risk for anemia grades 3-4 during the first year of ART.

\section{Discussion}

Our data from the African setting unveiled a complex interdependency between hepatotoxicity and anemia-inducing factors (e.g. HIV, type of NRTI, malaria) and -reducing factors such as increasing virologic suppression due to ART and a general improvement in health as a result of medical services. It is often problematic to compare the prevalence data and risk factors of liver toxicity and anemia from different studies because of varying definitions, grading and variability of transaminase or HB-determinations. Hepatotoxicity and anemia are multifactorial events, thus the study populations may differ in coinfections (TB, HBV, HCV), life styles (e.g. alcohol abuse), concomitant drug administration as well as in demographic and socio-economic variables.

The rate of pre-ART liver toxicity of any grade in our cohort was $18.6 \%$. That is higher than in industrialized countries, where $13.2 \%$ [18] to $15 \%$ [19] of HIV positive patients (but not co-infected with HBV or $\mathrm{HCV}$ ) show elevated transaminases. In African studies, the prevalence of pre-ART grade 1-4 liver toxicity ranges from $6.3 \%$ to $42 \%$ [5,20-23], and grades $3-4$ toxicity from $0.6 \%$ to $12.66 \%[5,21,24]$ whilst in our cohort it was $0.5 \%$.

The rate of pre-ART anemia grades 1-4 in our cohort was $35.2 \%$. Data from developed countries demonstrates considerable ranges from $1.3 \%$ to $95 \%[3,4]$, and from African cohorts other studies have demonstrated rates of $52.6 \%$ to $77.4 \%$ [25-27]. Our baseline pre-ART anemia levels were lower than that observed in other African cohorts.

\section{ART-associated hepatotoxicity and anemia during the first year of ART}

In order to analyze only ART-associated hepatotoxicity and anemia we excluded from further analysis all patients with abnormal transaminase and HB levels before the start of ART. Nevertheless, after the first month of ART we observed an elevated prevalence of hepatotoxicity grades 1-2 (13.5\%; d4T-arm: 14.6\%; AZT-arm: 9.1\%), hepatotoxicity grades $3-4(2.0 \%$; d4T: $1.9 \%$; AZT: $2.3 \%)$, anemia grades 1-2 (13.3\%; d4T: $12.1 \%$; AZT: $18.0 \%)$ and anemia grades $3-4(3.2 \%$; d4T: $3.2 \%$; AZT: $3.1 \%$ ). The prevalence of ART-induced liver toxicity grades 3-4 after one month in the cohort was four times higher than in ART-naïve patients (0.5\% vs. $2.0 \%)$. Other African studies report even

\begin{tabular}{|c|c|c|c|c|}
\hline & \multicolumn{2}{|c|}{ Univariate analysis } & \multicolumn{2}{|c|}{ Multivariate analysis } \\
\hline & \multicolumn{2}{|c|}{ Hazard ratio $(95 \% \mathrm{Cl})$} & \multicolumn{2}{|c|}{ Hazard ratio $(95 \% \mathrm{Cl})$} \\
\hline & Anemia 1-2 & Anemia 3-4 & Anemia 1-2 & Anemia 3-4 \\
\hline NRTI (d4T- vs. AZT) & $1.71(1.50-1.96)$ & $2.72(1.86-3.99)$ & $2.00(1.66-2.41)$ & $3.70(2.06-6.65)$ \\
\hline Pre-ART CD4+ cell count & Non-significant & Non-significant & Non-significant & Non-significant \\
\hline Pre-ART VL (>5.0 Log vs <5.0 Log) & Non-significant & $1.85(1.21-2.83)$ & Non-significant & $1.86(1.09-3.16)$ \\
\hline Pre-ART BMI (<18.5 vs. >18.6) & $0.75(0.64-0.89)$ & $0.43(0.26-0.70)$ & $0.71(0.59-0.86)$ & $0.43(0.25-0.73)$ \\
\hline Sex (female vs. male) & $1.46(1.40-1.56)$ & Non-significant & $2.22(1.84-2.68)$ & Non-significant \\
\hline Malaria & $1.29(1.07-1.54)$ & $2.21(1.40-3.52)$ & $1.28(1.01-1.63)$ & $4.05(2.30-7.12)$ \\
\hline
\end{tabular}

Table 5: Cox proportional hazards analysis of factors associated with ART-associated anemia grades 1-2 and 3-4. 
Citation: Wenderlein D, Scarcella P, Zimba I, Luhanga R, Mancinelli S, et al. (2016) Antiretroviral Treatment-Associated Hepatotoxicity and Anemia in Patients Receiving Stavudine or Zidovudine Containing Regimens in Sub-Saharan African Settings. J AIDS Clin Res 7: 537. doi:10.4172/21556113.1000537

Page 6 of 8

higher rates of early hepatotoxicity grades $3-4$ ranging between $1.3 \%$ and $14 \%$ ( 3 to 6 months after the initiation of ART) $[5,6,23,28]$.

The relatively high but brief increase in transaminase levels after the start of ART in patients who had no abnormal values before treatment shows the immediate livertoxic and anemic effect of d4T- and AZTcontaining regimens. These ART-associated toxicities are affected by other parameters such as HIV itself other diseases and drug-associated hepatotoxicities or anemia, probably combined with considerable intraindividual variability.

There is a declining trend of ART-associated hepatotoxicity and anemia over the first year of ART. This trend is more evident for anemia. The decline in liver toxicity prevalence starts immediately after one month of ART, but the decline in hepatotoxicity grades 1-2 is soon interrupted probably because of the strong hepatotoxic effect of d4T after 6 months of therapy. This is likely due to the mitochondrial liver toxicity of NRTIs, especially d4T, typically occurring at or after 6 months of ART $[8,9]$. Whether hepatotoxicity grades $1-2$ continues to decline after 9 to 12 months would be best answered by studies with longer follow up, even if the clinical relevance is questionable. Liver toxicity grades 3-4 declines to a minimum after 6 months of ART.

Data from patients in Malawi from comparable age and sex who did not take ART because they did not meet initiation criteria showed an average prevalence of $4.8 \%$ for hepatotoxicity grades $1-2$ and $0.8 \%$ for hepatotoxicity grades 3-4 (data not shown). These percentages are similar to our hepatotoxicity rates in the AZT-arm after three months of ART, which suggests that AZT does not contribute much to hepatotoxicity.

Studies analyzing early liver toxicity in cohorts from Uganda [23] and Nigeria [28] as well as long-term liver toxicity (24-36 months after start of ART) from Uganda [22,29] confirm a general decline of transaminases elevations during the course of ART. A South African study showed an increase of hepatotoxicity grades 1-2 in the first 3 months of ART from $9 \%$ to $15 \%$ and of hepatotoxicity grades 3-4 from $0.5 \%$ to $2 \%$ [5], however, in this trial, patients with pre-ART hepatotoxicity were not excluded. Studies from Uganda confirm a longterm stabilization of liver enzyme elevations at a prevalence of $13 \%$ after 24 months [29] and 12\% after 30 months post treatment initiation [22].

Studies from developed countries [3,4] and from Africa [26,30-33] show that HIV-associated anemia can be reversed by ART, but even after 12 months a portion of patients remain anemic. Our data confirms the immediate and lasting reduction of ART-associated anemia grades 1-4 through the first year of ART. Anemia reducing factors due to HIV treatment outbalance inducing factors by far. Most likely the fraction of AZT-associated anemia which exceeds the anemia found in the d4Tarm is the only truly ART-attributable anemia.

\section{Risk factors for hepatotoxicity and anemia}

In our cohort, the strongest risk factor for ART-associated hepatotoxicity grades 1-2 during the first year of ART was d4T, but the use of $\mathrm{d} 4 \mathrm{~T}$ was not associated with hepatotoxicity grades $3-4$. Increased pre-ART VL and female gender were risk factors for mild to moderate liver toxicities as well. A good nutritional status at the start of ART was protective against hepatotoxicty grades $1-2$. We could not find significant risk factors for hepatotoxicity grades $3-4$.

The association between hepatotoxicity and gender is conflicting in other African studies: a South African cohort confirmed female gender as a risk factor [6], while two Ugandan studies identified male gender as a risk factor [22,29].Two other South African studies did not find any association between sex and hepatotoxicity $[5,21]$.
Cohort studies from South Africa [6] and Uganda [29] confirm our findings that a good nutritional status protects against early hepatotoxicity, while in developed countries being overweight is associated with elevated transaminases [18,34]. Probably a normal body weight limits ART-associated hepatotoxicity.

Interestingly, in our cohort the pre-ART CD4 count was of minor importance as a risk factor for liver toxicity: only in the univariate analysis a low pre-ART CD4 count was associated with hepatotoxicity grades 1-2. There are concerns that elevated CD4 counts may be associated with NVP-induced liver toxicities, but data are conflicting $[6,35]$. Because of that WHO treatment guidelines from 2013 [14] suggest that NVP should be used with caution in women with higher CD4 counts and in pregnant women. Data from a Malawian cohort of HIV-infected, NVP-treated pregnant women in the DREAM Program [36] show that baseline levels of CD4 $>250$ cells/ $\mu$ l were indeed correlated with early moderate or severe (grade $\geq 2$ ) ALT elevations. But because of the relatively low overall incidence of moderate to severe liver toxicity (9\%) in the long-term follow-up the authors suggest that the use of NVP in this context is relatively safe [37].

AZT and cumulative malaria are the strongest risk factors for anemia of any grade in the first year of ART. As for hepatotoxicity grades 1-2, a good nutritional condition protects against anemia. Patients with a poor health status at the initiation of ART (high VL, malnutrition, malaria) are especially at risk of developing ART-associated toxicities like anemia, in particular severe and life threatening anemia, and hepatotoxicity grades 1-2.

The few African studies which identified risk factors for anemia in patients on ART are conflicting because of different study conditions and populations. In a cohort from Rwanda [33], risk factors in uniand multivariate analysis were AZT, a low BMI, lack of ART, a CD4 count below 200 and TB. A cohort from Côte d'Ivoire [30] analyzing age, VL, CD4 count, ALT/AST, pre-ART anemia 1-2, WHO stage and $\mathrm{BMI}$ found a pre-ART HB between 8 and $10.5 \mathrm{~g} / \mathrm{dl}$ as the only risk factor for anemia grades 3-4. A Tanzanian cohort identified AZT as risk factor compared to d4T [26]. The studies did not exclude patients with preART anemia.

\section{Nevirapine versus efavirenz}

NVP causes early hepatotoxicity (within 1 to 8 weeks) due to hypersensitivity reactions and late idiosyncratic hepatotoxicity (2 to 6 months) [1]. NVP is associated with a higher rate of hepatotoxicity compared to EFV [6,34,37,38]. Our objective to evaluate NVP compared to EFV as a risk factor could not be achieved because the number of patients who received EFV was too small to reach statistical significance, despite of the large sample size. A repeat analysis excluding patients who received EFV did not change the results.

\section{Limitations of the study}

This study has some limitations: $\mathrm{TB}$, concomitant TB-treatment [21], HCV and HBV [39] may cause liver enzymes elevations in HIV infected persons, but we could not evaluate their influence on liver toxicity because this data was not registered in the software for TB and due to the lack of diagnostic means for hepatitis viruses.

In a Malawian cohort of HIV-infected pregnant women, 28 out of 309 women $(9.1 \%)$ were co-infected with either HBV $(8.7 \%, n=27)$ or HCV $(0.3 \%, n=1)$. During 2 years of follow-up $40.4 \%$ developed liver toxicity of any grade, $9.1 \%$ had moderate to severe liver toxicity (grade $\geq 2$ ) and $1.9 \%$ had grade 3 liver toxicity [36]. 
Citation: Wenderlein D, Scarcella P, Zimba I, Luhanga R, Mancinelli S, et al. (2016) Antiretroviral Treatment-Associated Hepatotoxicity and Anemia in Patients Receiving Stavudine or Zidovudine Containing Regimens in Sub-Saharan African Settings. JAIDS Clin Res 7: 537. doi:10.4172/21556113.1000537

Page 7 of 8

In a multivariate model either $\mathrm{HBV}$ or $\mathrm{HCV}$ infection were significantly associated with the development of mild liver toxicity. The only predictor of moderate or severe hepatotoxicity (grade $\geq 2$ ) was a baseline CD4 cell count $>250 / \mu$ l. Nearly half of the cases $(46.4 \%)$ of grade $\geq 2$ toxicity developed within the first 18 weeks of treatment. The presence of HBV or HCV infection did not seem to have a major impact on the emergence of moderate to severe liver toxicities.

We had a proportion of patients with incomplete monitoring of AST, HB, CD4, VL and BMI. The reason that not all data points were available for analysis is that data collection was done under real world conditions, including patient with insufficient retention, and noncollection of specific measures during clinic visits.

\section{Conclusion}

This observational study shows that in sub-Saharan Africa, the presence of hepatotoxicity grades 1-2 is a significant adverse effect of the use of $\mathrm{d} 4 \mathrm{~T}$, and anemia of any grade is associated with AZT use during the first year of ART. Patients with poor health status at the initiation of ART as in the case of malnutrition, an increased VL and/ or malaria have an increased risk of developing ART related side effects, especially anemia. African risk factors like malaria and malnutrition have to be treated also in order to reduce side effects. Baseline CD4 is of less importance in this context. ART-associated mortality was low. Less toxic NRTIs and modern protease-, integrase- and entry inhibitors should be made available in Africa in order to reduce this toxicity profile.

There is a need to standardize the definitions of hepatotoxicity and anemia in order to improve comparability of results. Frequent, regular routine monitoring of liver enzymes and $\mathrm{HB}$ screening before and during ART are recommended in order to identify HIV patients at highest risk of disease progression and death.

\section{Competing Interests}

The authors declare that they have no competing interests.

\section{Acknowledgement}

The authors acknowledge all study participants, the multiple members of the DREAM program who participated in this study, and especially the DREAM coordinators in Mozambique and Malawi. We thank Prof. Claudia Martini, Prof. Adriano Martinelli and Prof. Federico Da Settimo Passetti from the Faculty of Pharmacy of the University of Pisa, Italy, for facilitating the study.

\section{References}

1. Soriano V, Puoti M, Garcia-Gascó P, Rockstroh JK, Benhamou Y, et al. (2008) Antiretroviral drugs and liver injury. AIDS 22: 1-13.

2. Núñez M (2010) Clinical syndromes and consequences of antiretroviral-related hepatotoxicity. Hepatology 52: 1143-1155.

3. Belperio PS, Rhew DC (2004) Prevalence and outcomes of anemia in individuals with human immunodeficiency virus: a systematic review of the literature. Am J Med 116 Suppl 7A: 27S-43S.

4. Harris RJ, Sterne JA, Abgrall S, Dabis F, Reiss P, et al. (2008) Prognostic importance of anaemia in HIV type-1-infected patients starting antiretroviral therapy: collaborative analysis of prospective cohort studies. Antivir Ther 13 959-967.

5. Chu KM, Boulle AM, Ford N, Goemaere E, Asselman V, et al. (2010) Nevirapineassociated early hepatotoxicity: incidence, risk factors, and associated mortality in a primary care ART programme in South Africa. PLoS One 5: e9183.

6. Sanne I, Mommeja-Marin H, Hinkle J, Bartlett JA, Lederman MM, et al. (2005) Severe hepatotoxicity associated with nevirapine use in HIV-infected subjects. $\mathrm{J}$ Infect Dis 191: 825-829.

7. Montessori V, Harris M, Montaner JS (2003) Hepatotoxicity of nucleoside reverse transcriptase inhibitors. Semin Liver Dis 23: 167-172.

8. Ogedegbe AO, Sulkowski MS (2003) Antiretroviral-associated liver injury. Clin Liver Dis 7: 475-499.
9. Dieterich DT, Robinson PA, Love J, Stern JO (2004) Drug-induced liver injury associated with the use of nonnucleoside reverse-transcriptase inhibitors. Clin Infect Dis 38 Suppl 2: S80-89.

10. Marazzi MC, Liotta G, Germano P, Guidotti G, Altan AD, et al. (2008) Excessive early mortality in the first year of treatment in HIV type 1-infected patients initiating antiretroviral therapy in resource-limited settings. AIDS Res Hum Retroviruses 24: 555-60

11. May M, Boulle A, Phiri S, Messou E, Myer L, et al. (2010) Prognosis of patients with HIV-1 infection starting antiretroviral therapy in sub-Saharan Africa: a collaborative analysis of scale-up programmes. Lancet 376: 449-457.

12. DeJesus E, Herrera G, Teofilo E, Gerstoft J, Buendia CB, et al. (2004) Abacavir versus zidovudine combined with lamivudine and efavirenz, for the treatment of antiretroviral-naive HIV-infected adults. Clin Infect Dis 39: 1038-1046.

13. Gallant JE, DeJesus E, Arribas JR, Pozniak AL, Gazzard B, et al. (2006) Tenofovir DF, emtricitabine, and efavirenz vs. zidovudine, lamivudine, and efavirenz for HIV. N Engl J Med 354: 251-260.

14. WHO Guidelines (2013) Consolidated guidelines on the use of antiretroviral drugs for treating and preventing HIV infection.

15. AIDS Clinical Trials Group Division of AIDS (2009) Table for grading the severity of adult and pediatric adverse events. Version 1.0

16. Haldar K, Mohandas N (2009) Malaria, erythrocytic infection, and anemia Hematology Am Soc Hematol Educ Program.

17. Sheehy SH, Angus BJ (2011) Malaria: severe, life-threatening. BMJ Clin Evid 2011.

18. Kovari H, Ledergerber B, Battegay M, Rauch A, Hirschel B, et al. (2010) Incidence and risk factors for chronic elevation of alanine aminotransferase levels in HIV-infected persons without hepatitis b or $c$ virus co-infection. Clin Infect Dis 50: 502-511.

19. Sterling RK, Chiu S, Snider K, Nixon D (2008) The prevalence and risk factors for abnormal liver enzymes in HIV-positive patients without hepatitis B or C coinfections. Dig Dis Sci 53: 1375-1382.

20. Weidle PJ, Malamba S, Mwebaze R, Sozi C, Rukundo G, et al. (2002) Assessment of a pilot antiretroviral drug therapy programme in Uganda: patients' response, survival, and drug resistance. Lancet 360: 34-40.

21. Hoffmann CJ, Charalambous S, Thio CL, Martin DJ, Pemba L, et al. (2007) Hepatotoxicity in an African antiretroviral therapy cohort: the effect of tuberculosis and hepatitis B. AIDS 21: 1301-1308.

22. Ocama P, Castelnuovo B, Kamya MR, Kirk GD, Reynolds SJ, et al. (2010) Low frequency of liver enzyme elevation in HIV-infected patients attending a large urban treatment centre in Uganda. Int J STD AIDS 21: 553-557.

23. Kalyesubula R, Kagimu M, Opio KC, Kiguba R, Semitala CF, et al. (2011) Hepatotoxicity from first line antiretroviral therapy: an experience from a resource limited setting. Afr Health Sci 11: 16-23.

24. Goni BW, Yusuph H, Mustapha SK, Sahabi MA, Gwalabe SA et al. (2013) Hepatic transaminase and alkaline phosphatase enzyme levels in HIV/HBV coinfected and HIV mono-infected patients in Maiduguri, Nigeria. Niger J Clin Pract 16: 530-534

25. Omoregie R, Omokaro EU, Palmer O, Ogefere HO, Egbeobauwaye A, et al. (2009) Prevalence of anaemia among HIV-infected patients in Benin City, Nigeria. Tanzan J Health Res 11: 1-4

26. Johannessen A, Naman E, Gundersen SG, Bruun JN (2011) Antiretrovira treatment reverses HIV-associated anemia in rural Tanzania. BMC Infect Dis 11: 190

27. Adane A1, Desta K, Bezabih A, Gashaye A, Kassa D (2012) HIV-associated anaemia before and after initiation of antiretroviral therapy at Art Centre of Minilik II Hospital, Addis Ababa, Ethiopia. Ethiop Med J 50: 13-21.

28. Ugiagbe RA1, Ugiagbe EE (2012) Period of onset and lack of clinical manifestation of hepatotoxicity after commencing highly active antiretroviral therapy. Niger J Clin Pract 15: 63-67.

29. Weidle PJ, Moore D, Mermin J, Buchacz K, Were W, et al. (2008) Live enzymes improve over twenty-four months of first-line non-nucleoside reverse transcriptase inhibitor-based therapy in rural Uganda. AIDS Patient Care STDS 22: 787-95.

30. Moh R, Danel C, Sorho S, Sauvageot D, Anzian A, et al. (2005) Haematologica 
Citation: Wenderlein D, Scarcella P, Zimba I, Luhanga R, Mancinelli S, et al. (2016) Antiretroviral Treatment-Associated Hepatotoxicity and Anemia in Patients Receiving Stavudine or Zidovudine Containing Regimens in Sub-Saharan African Settings. JAIDS Clin Res 7: 537. doi:10.4172/21556113.1000537

changes in adults receiving a zidovudine-containing HAART regimen in combination with cotrimoxazole in Côte d'Ivoire. Antivir Ther 10: 615-624.

31. Forna F, Moore D, Mermin J, Brooks JT, Were W, et al. (2009) Hematologic changes associated with Zidovudine following single-drug substitution from stavudine in a home-based AIDS care program in rural Uganda. $\mathrm{J}$ Int Assoc Physicians AIDS Care 8: 128-38.

32. Kiragga AN, Castelnuovo B, Nakanjako D, Manabe YC (2010) Baseline severe anaemia should not preclude use of zidovudine in antiretroviral-eligible patients in resource-limited settings. J Int AIDS Soc 13: 42.

33. Masaisa F, Gahutu JB, Mukiibi J, Delanghe J, Philippé J (2011) Anemia in human immunodeficiency virus-infected and uninfected women in Rwanda. Am J Trop Med Hyg 84: 456-460.

34. Sulkowski MS, Mehta SH, Torbenson M, Afdhal NH, Mirel L, et al. (2005) Hepatic steatosis and antiretroviral drug use among adults co-infected with HIV and hepatitis C virus. AIDS 19: 585-592.

35. Manfredi R, Calza L (2006) Nevirapine versus efavirenz in 742 patients: no link of liver toxicity with female sex, and a baseline CD4 cell count greater than 250 cells/microl. AIDS 20: 2233-2236.

36. Andreotti M, Pirillo MF, Liotta G, Jere H, Maulidi M, et al. (2014) The impact of $\mathrm{HBV}$ or HCV infection in a cohort of HIV-infected pregnant women receiving a nevirapine-based antiretroviral regimen in Malawi. BMC Infect Dis 14: 180

37. Marazzi MC, Germano P, Liotta G, Guidotti G, Loureiro S, et al. (2006) Safety of nevirapine- containing antiretroviral triple therapy regimens to prevent vertical transmission in an African cohort of HIV-1-infected pregnant women. HIV Med 7:338-344.

38. van Leth F, Phanuphak P, Ruxrungtham K, Baraldi E, Miller S, et al. (2004) Comparison of first-line antiretroviral therapy with regimens including nevirapine efavirenz, or both drugs, plus stavudine and lamivudine: a randomised openlabel trial, the 2NN Study. Lancet 363: 1253-1263.

39. Mbougua JB, Laurent C, Kouanfack C, Bourgeois A, Ciaffi L, et al. (2010) Hepatotoxicity and effectiveness of a Nevirapine-based antiretroviral therapy in HIV-infected patients with or without viral hepatitis B or C infection in Cameroon. BMC Public Health 